Research Article

\title{
Performance Analysis of a Kretschmann-Based Ag-ITO-Au Surface Plasmon Resonance Sensor through Numerical Simulations
}

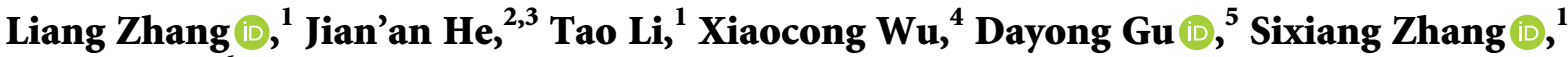 \\ and Ying $\mathbf{Y e}^{6}$ \\ ${ }^{1}$ School of Mechanical Engineering, Hebei University of Technology, Tianjin 300401, China \\ ${ }^{2}$ Central Laboratory of Health Quarantine, Shenzhen International Travel Health Care Center, Shenzhen, China \\ ${ }^{3}$ Shenzhen Academy of Inspection and Quarantine, Shenzhen Customs District, Shenzhen 518000, China \\ ${ }^{4}$ School of Public Health, Guangdong Medical University, Dongguan 523808, China \\ ${ }^{5}$ Department of Clinical Laboratory, Shenzhen Institute of Translational Medicine, \\ The First Affiliated Hospital of Shenzhen University, Shenzhen Second People's Hospital, Shenzhen 518000, China \\ ${ }^{6}$ Shenzhen International Travel Health Care Center, Shenzhen Customs, Shenzhen 518000, China
}

Correspondence should be addressed to Dayong Gu; wanhood@163.com and Sixiang Zhang; zhangsx@hebut.edu.cn

Received 27 March 2021; Revised 20 November 2021; Accepted 26 November 2021; Published 22 December 2021

Academic Editor: Giancarlo C. Righini

Copyright (c) 2021 Liang Zhang et al. This is an open access article distributed under the Creative Commons Attribution License, which permits unrestricted use, distribution, and reproduction in any medium, provided the original work is properly cited.

\begin{abstract}
Variations of a Kretschmann-structure-based Ag-indium tin oxide- (ITO-) Au surface plasmon resonance (SPR) sensor were explored to improve its sensitivity. The sensor structure was optimised, and its characteristics were studied through numerical simulations. The chip structure that comprised $20 \mathrm{~nm} \mathrm{Ag} / 30 \mathrm{~nm}$ ITO/10 $\mathrm{nm}$ Au yielded the best sensing performance, wherein the angular sensitivity could reach $197.6^{\circ} \mathrm{RIU}^{-1}$ and the figure of merit was $43.4 \mathrm{RIU}^{-1}$. These performance parameters are nearly three times higher than those of $\mathrm{Ag} / \mathrm{Au}$ bimetallic resonance sensors. Furthermore, an adhesive $\mathrm{Cr}$ layer and two-dimensional graphene were incorporated into this sensor structure to explore their impact on the performance. The results demonstrated that the $\mathrm{Cr}$ layer significantly weakened the sensor performance, whereas graphene did not produce the expected enhancement effect on this structure. If simply adding a layer to a $\mathrm{Au} / \mathrm{Ag}$ sensor can produce a three-fold improvement in its performance, then its economic and scientific benefits are potentially significant and widespread.
\end{abstract}

\section{Introduction}

Surface plasmon resonance (SPR) is a technique for detecting changes in refractive index of matter via the photoelectric effect. Theoretically, any phenomenon that causes a change in the refractive index can be monitored and recorded using SPR. Therefore, SPR sensors are commonly used in food, medicine, biomedicine, and gas detection for their high sensitivity $[1,2]$. Also, the sensor chip is extremely significant and widely researched as it is the main part involved in resonance and detection. The interior of a metal and its surface contain abundant free electrons. These electrons which move under the action of Coulomb forces are called surface plasmon waves (SPWs). SPWs propagate along the metal surface and decay exponentially in the direction perpendicular to the surface $[3,4]$. If a beam of P-polarized light is incident on the metal surface, resonance can occur when the wave vectors are equal in size, direction, and angular frequency $[5,6]$. When resonance occurs, the incident light wave is strongly absorbed, and the reflected light is clearly weakened. Consequently, the stronger the resonance, the quicker the response of the sensor chip to the change in the refractive index based on light wave absorption, which is one of the most common and effective ways to improve the sensitivity of an SPR sensor system. 
SPR sensors are extensively used in many fields, but because of their sensitivity problems, the detection of some small-molecular-weight substances is not very accurate. Many studies have attempted to improve the sensitivity of SPR sensors by reconstructing the sensor chips. For the first time, Cai et al. [7] reported the systematic construction and evaluation of a graphene-modified gold-plated film chip. By comparing the simulation and experimental results (by Winspall simulation software), they discovered that each layer of graphene could cause a resonance angle offset of approximately $0.18^{\circ}$. Meanwhile, Verma et al. [8] added a silicon layer with a higher refractive index between the gold film and graphene layer and developed a sensor comprising a $40 \mathrm{~nm}$-thick gold layer, a $7 \mathrm{~nm}$-thick silicon layer, and two layers of graphene with a sensitivity more than twice that of traditional gold films. Zhao et al. [9] added transition-metal dichalcogenides on both sides of the metal, causing the angle sensitivity of the SPR chip to reach $315.5^{\circ} \mathrm{RIU}^{-1}$; however, because of its large resonance angle and half peak width, its figure of merit (FOM) is only 18.8, which is relatively low. Gan et al. [10] added indium tin oxide (ITO) conductive glass, a new electronic material, to a sensing chip composed of chromium $\mathrm{Cr}$ and $\mathrm{Ag}$. The experimental results show that the chip angle sensitivity can reach $68.77^{\circ} \mathrm{RIU}^{-1}$. Furthermore, the FOM is 11.26 for the $0.5 \mathrm{~nm} \mathrm{Cr} / 30 \mathrm{~nm} \mathrm{Ag} / 10 \mathrm{~nm}$ ITO combination, wherein the resonance angle is approximately $43^{\circ}$ and the minimum light reflectivity is approximately $9.03 \%$. Therefore, ITO has proved effective for improving chip sensitivity.

Thus, extending the results of previous studies, an $\mathrm{Ag} / \mathrm{ITO} / \mathrm{Au}$ laminated SPR biosensor chip based on the Kretschmann structure, shown in Figure 1, was developed in this study. Although the Ag layer has better resonance than the Au layer, the chemical properties of Ag make it unstable and easily oxidisable [11], and therefore, it is present at the bottom of the newly proposed structure as the base layer. The ITO layer insulates Ag from Au; it can transmit SPWs fast enough to enhance resonance. Because of its low resistivity, strong ultraviolet absorption, high transmittance in the visible light region, and high reflectivity in the far-infrared region, ITO is widely used as a thin transparent coating in sensors and on electrodes. Nevertheless, because the surface of ITO is characterised by strong water absorption, it can easily form 'mildew' on reacting with water and carbon dioxide in the air; thus, a thin layer of chemically stable Au is normally coated onto the surface of ITO as a protective layer to protect it from moisture in the air. In contrast, as a kind of III-V semiconductor oxide [12], ITO can stop the resonances of $\mathrm{Ag}$ and $\mathrm{Au}$ from affecting each other if the three materials form the sandwich structure shown in Figure 1. Owing to the good conductivity of ITO, the resonance SPWs formed at the interface can be quickly transmitted outward. Because the outermost ITO layer is covered by the Au film, traditional chemical modification methods can still be used to fix the biosensitive layer.

In this study, the COMSOL finite-element analysis software was used to solve Maxwell's equations, and the optimum $\mathrm{Ag} / \mathrm{ITO} / \mathrm{Au}$ structure was determined. In addition, the enhancement effect of graphene $(G)$ layers on the resonance when placed at different positions was explored. Furthermore, the effect of $\mathrm{Cr}$ layers with different thicknesses on the sensing chip was analysed via numerical simulation. Finally, the performance parameters of the sensor were evaluated.

\section{Method and Model Parameters}

SPR is mainly used to explore the influence of an electromagnetic field on electron motion at the microscale. This process can be described using Maxwell's partial differential equations. The main electromagnetic simulation methods are the finite-difference time-domain method (FDTD), which can be conducted using the software FDTD Solutions, and the finite-element method (FEM), which can be performed using software packages such as HFSS and COMSOL. FDTD is an iterative method that begins the calculation from one source point. This method is quick, but its simple mesh division produces inaccurate results. Moreover, the entire mesh structure in FDTD can be discredited by FEM, wherein a complex structure can be divided arbitrarily. However, in FEM, a simple method is used to approximate a complex problem, and an approximate solution is used to determine the real solution. The calculated results are more accurate, but the method is slow and consumes more memory. This study required a more accurate FEM to solve the Maxwell equation using COMSOL. The radio-frequency (rf) module in COMSOL can be used to calculate the electric and magnetic fields of high-frequency systems. Total fields or scattering fields can be solved using the time harmonic and transient electromagnetic fields in the full vector formula.

As SPR has no bearing on the 3D scale, a $2 \mathrm{D}$ model was built. Part of the model that was comparable to the wavelength size was selected for analysis and was set to Floquet periodicity. The laminated sensor was scheduled to operate at a wavelength of $670 \mathrm{~nm}$ to reduce the impact of the stabilised Cr layer. At this wavelength, the refractive indices of $\mathrm{Ag}$, ITO, and $\mathrm{Au}$ are $0.1783+4.7096 i[10], 1.7450+0.0036 i[10]$, and $0.161+3.4458 i$ (date from Filmetrics Inc., USA, https://www.filmetrics.cn/ refractive-index-database/Au/Gold), respectively; these parameters were manually configured in the parameter bar. The model of this laminated structure attached to a BK7 glass substrate $(n=1.5202)$ is illustrated in Figure 2. Beginning from the top, the layers in the diagram represent glass $(500 \mathrm{~nm}), \mathrm{Ag}$ (variable), ITO (variable), Au (variable), and air (500 nm; $n=1)$. The free triangle mesh was used for division, and the minimum element size was $0.08 \mathrm{~nm}$. Changes in the incident angle in the $0-90^{\circ}$ range were used for parameter scanning. The direct solver solution was applied to obtain the general reflectivity curve. The PC required approximately $80-100 \mathrm{~min}$ to process each set of data. Finally, the effects of the $\mathrm{Cr}$ and graphene layers on the sensor performance were studied, and the sensor performance was evaluated against biological solutions with different refractive indices.

\section{Results and Discussion}

Generally, the smaller the strongest resonance angle, the larger the range of changes that can occur in the resonance angle (up to $90^{\circ}$ ); thus, changes in refractive index can be 


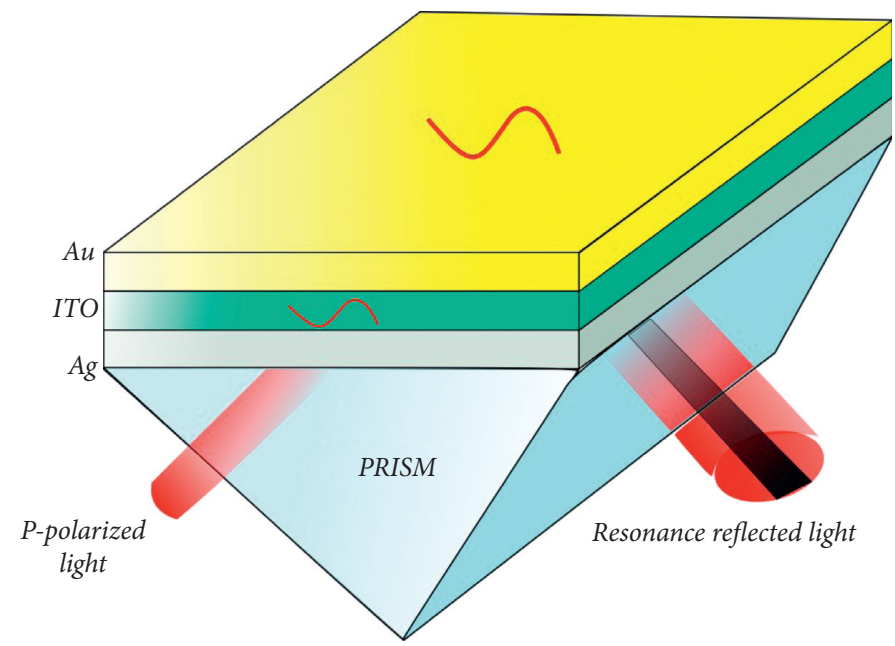

FIGURE 1: SPR schematic and chip structure.

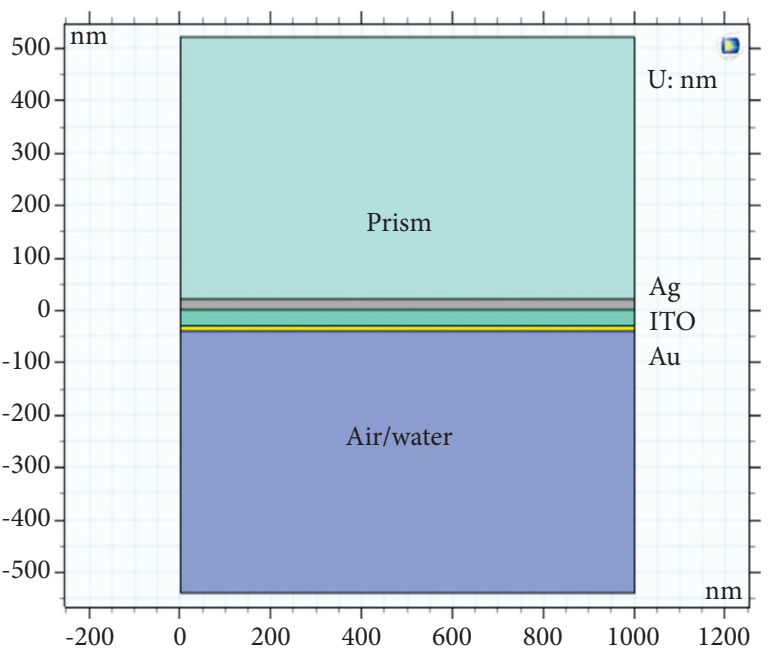

Figure 2: 2D model diagram.

detected to a higher extent. A lower reflection signal at the strongest resonance angle indicates that the higher the light energy absorbed by the surface plasma, the stronger the resonance and better the SPR sensing effect; however, this is not the main criterion for evaluating the sensor performance. There are many other evaluation criteria, such as sensitivity, full width at half maximum (FWHM), and FOM. The latter is an extremely important parameter for evaluating the performance of the entire chip; the larger the FOM, the better the performance of the chip. Sensitivity $(S)$ is described as the change in the resonance angle $\left(\Delta \theta_{\text {res }}\right)$ per refractive index unit (RIU), i.e., $S=\left(\Delta \theta_{\text {res }} / \Delta n\right) \mathrm{RIU}^{-1}$. FWHM refers to the peak width at the midpoint of the resonance peak. FOM refers to the ratio of $S$ to FWHM, i.e., $\mathrm{FOM}=S / \mathrm{FWHM}=\left(\Delta \theta_{\text {res }} / \Delta n\right) / \mathrm{FWHM}[13-15]$. From the definition of FOM, we can infer that the higher the sensitivity of the SPR chip, the smaller the FWHM, the larger the corresponding FOM, and the better the quality. In this study, the minimum $S$ and FOM were used as the criteria for comprehensive evaluation of the chip performance.
3.1. Determination of Film Thickness. To obtain the best resonance effect, different film thickness combinations were numerically simulated. For conducting the simulation, the thickness of the outermost gold film was first fixed at $10 \mathrm{~nm}$, and then, the thicknesses of Ag and ITO were varied. The variation range was $10-50 \mathrm{~nm}$. The results are shown in Figures 3(a)-3(e). The red line represents the best light absorption exhibited by the surface plasma for each combination. The analysis revealed that $\theta_{\text {res }}$ increased with the increase in thickness of the Ag film. Also, the minimum resonance peak of the SPR signal increased, and the FWHM increased. Moreover, as the thickness of the ITO film layer increased, $\theta_{\text {res }}$ increased, and the resonance peak decreased in size. When the Ag film thickness was $20 \mathrm{~nm}$, ITO exhibited considerably low reflectivity, with the following values obtained in the thickness range of $10-50 \mathrm{~nm}: 1.38 \%$ $\left(\mathrm{FWHM}=2.31, \quad \theta_{\text {res }}=43.8^{\circ}\right), \quad 0.56 \% \quad(\mathrm{FWHM}=2.27$, $\left.\theta_{\text {res }}=44.2^{\circ}\right), \quad 0.08 \% \quad\left(\mathrm{FWHM}=2.04, \quad \theta_{\text {res }}=44.6^{\circ}\right), \quad 0.19 \%$ $\left(\mathrm{FWHM}=2.30, \quad \theta_{\text {res }}=45^{\circ}\right)$, and $0.36 \% \quad(\mathrm{FWHM}=2.59$, $\theta_{\text {res }}=45.6^{\circ}$ ). When the thickness of the Ag and ITO layers was 20 and $30 \mathrm{~nm}$, respectively, the model structure almost completely absorbed the incident light waves. Table 1 shows these values. To determine whether this structure could be affected by the thickness of the ITO layer, the ITO thickness was increased to $120 \mathrm{~nm}$, wherein the resonance intensity, resonance angle, and FWHM of the SPR signal exhibited obvious changes, as indicated by the green curve in Figure 3(b).

The optimal thicknesses of the Ag and ITO layers were determined, and the thickness of the outermost Au film was varied as the simulation continued. Figure 4 shows the effect of changing the thickness of the Au film from 10 to $50 \mathrm{~nm}$ on the Ag/ITO chip combination. The resonance peak of the chip continued to increase significantly, whereas FWHM continued to decrease as the Au layer became thicker. After processing all the collected data, the optimal thicknesses were determined to be $20 \mathrm{~nm}$ for $\mathrm{Ag}$, $30 \mathrm{~nm}$ for ITO, and $10 \mathrm{~nm}$ for $\mathrm{Au}$ for the laminated Ag/ITO/Au structure. The Ag base layer mainly changes the resonance peak size at thicknesses above $20 \mathrm{~nm}$, and the 


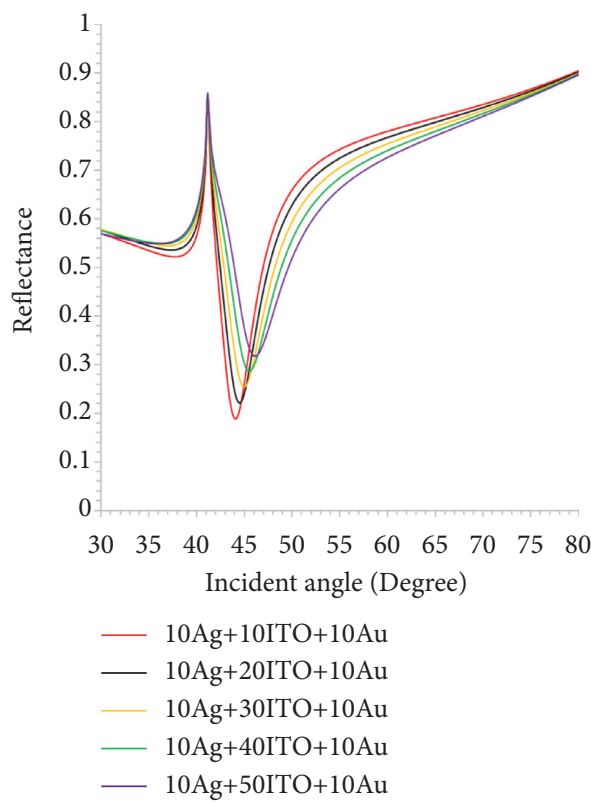

(a)

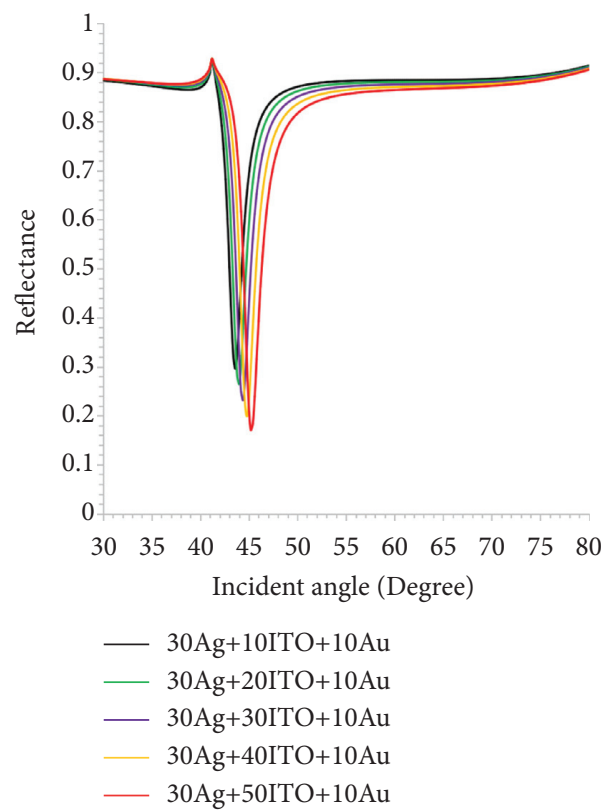

(c)

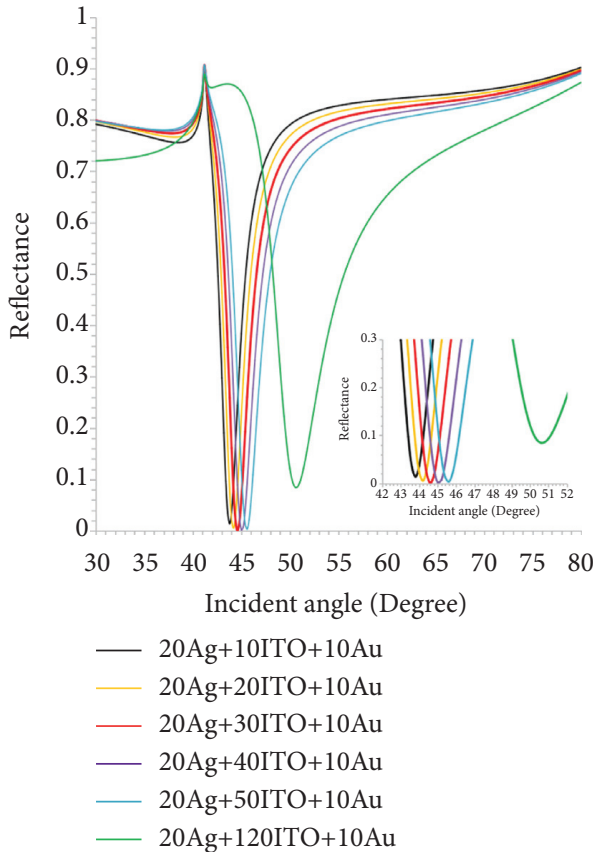

(b)

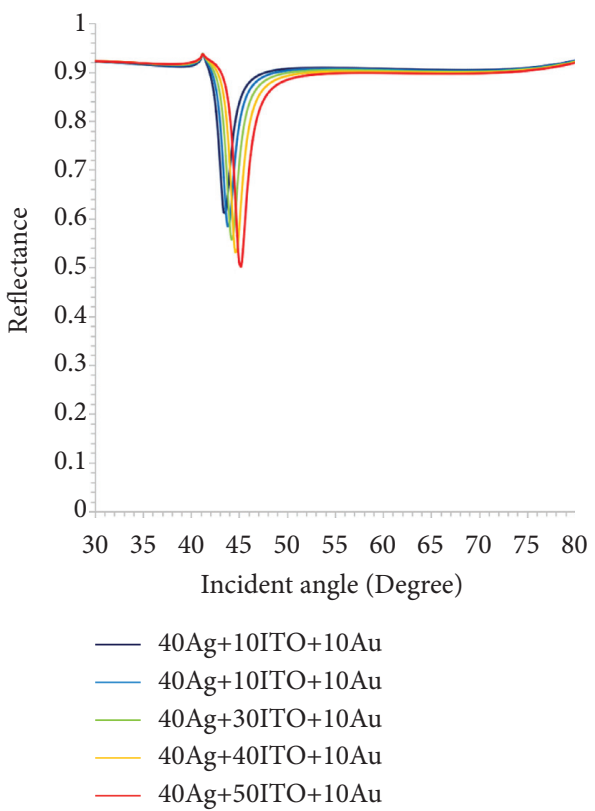

(d)

Figure 3: Continued. 


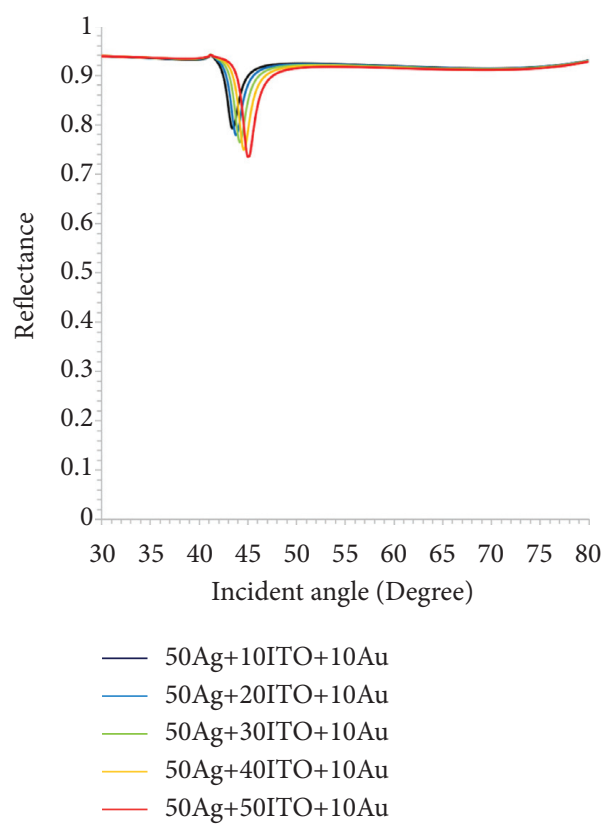

(e)

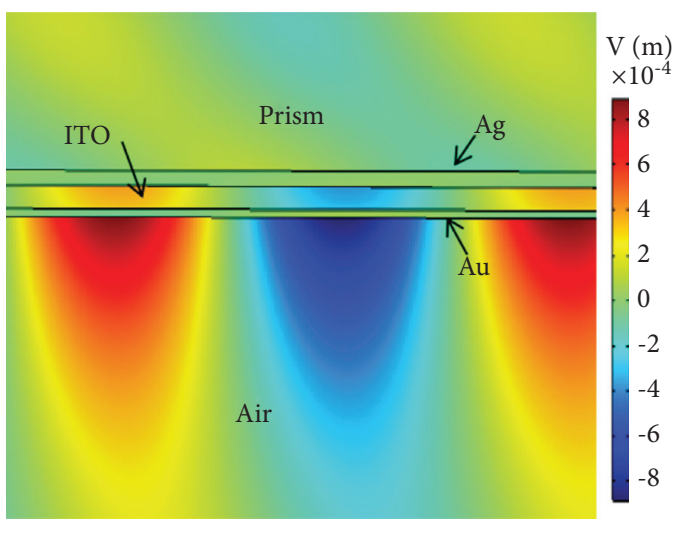

(f)

FIGURE 3: Changes in chip reflectivity when thicknesses of Ag and ITO change from 10 to 50; the red line represents the best structure; the green line in (b) represents the reflectivity of the chip when ITO thickness is 120; (f) the electromagnetic field distribution corresponding to the structure represented by the red line in (b) (unit: $\mathrm{nm}$ ).

TABLE 1: Reflectivity changes caused by ITO with different thicknesses.

\begin{tabular}{lccc}
\hline ITO thickness $(\mathrm{nm})$ & $R_{\min }(\%)$ & FWHM & $\theta_{\text {res }}$ \\
\hline 10 & 1.38 & 2.31 & $43.8^{\circ}$ \\
20 & 0.56 & 2.27 & $44.3^{\circ}$ \\
30 & 0.08 & 2.04 & $44.6^{\circ}$ \\
40 & 0.19 & 2.30 & $45.0^{\circ}$ \\
50 & 0.36 & 2.59 & $45.6^{\circ}$ \\
\hline
\end{tabular}

ITO interlayer causes the resonance angles to shift toward the left and right; the Au film as the outermost layer can reduce the FWHM when its thickness exceeds $10 \mathrm{~nm}$. To further improve the sensing chip performance, the effect of adding graphene to the $20 \mathrm{~nm} \mathrm{Ag/30} \mathrm{nm} \mathrm{ITO/10} \mathrm{nm} \mathrm{Au}$ stacked structure was investigated.

3.2. Effects of Graphene $(G)$ on Chip. A graphene monolayer is a honeycomb-like two-dimensional crystalline material comprising carbon atoms with excellent optical and electrical conductivities. Its surface is rich in electrons and can enhance the propagation constant of surface plasma. Graphene can improve the performance of SPR sensors [18-20]. The thickness of a graphene monolayer is only $0.34 \mathrm{~nm}$, and its transmittance can reach $97.7 \%$. The complex refractive index of the graphene monolayer is calculated as $n_{G}=3+i C / 3 \lambda$, where $C$ is a constant equal to $5.446 \mu \mathrm{m}^{-1}$ and $\lambda$ is the wavelength [21]. To develop the best chip structure, the graphene monolayer was placed at different positions in the laminated chip to explore its effect on the chip performance.

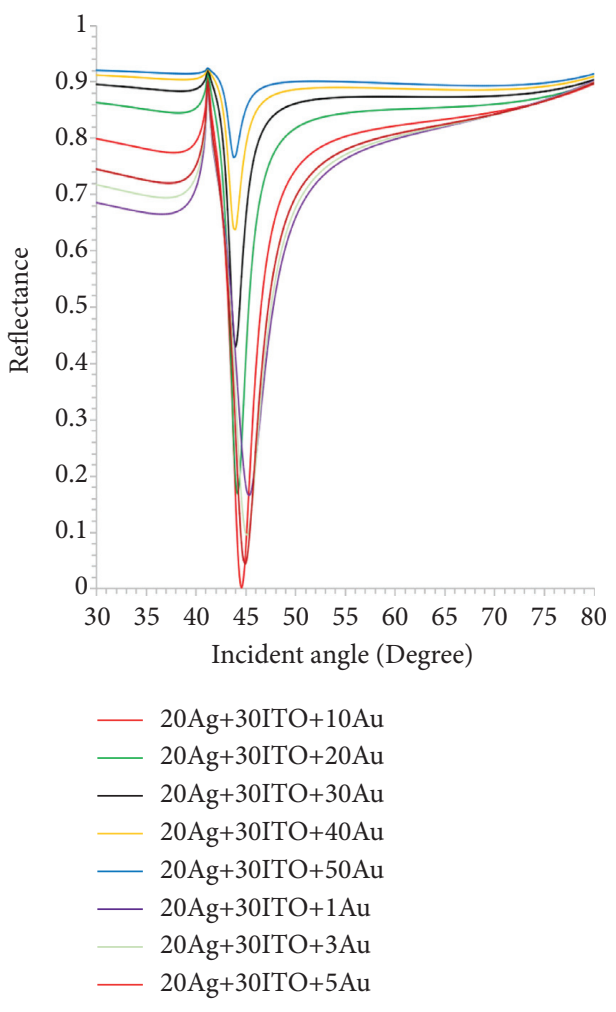

FIGURE 4: Minimum reflectivity of the sensor when the outermost $\mathrm{Au}$ layer thickness changes from 1 to 10 (unit: $\mathrm{nm}$ ).

Monolayer and bilayer graphene were placed in several combinations on both sides of the ITO and Au layers are shown in Figure 5. The black line in Figures 5(a) and 5(b) 


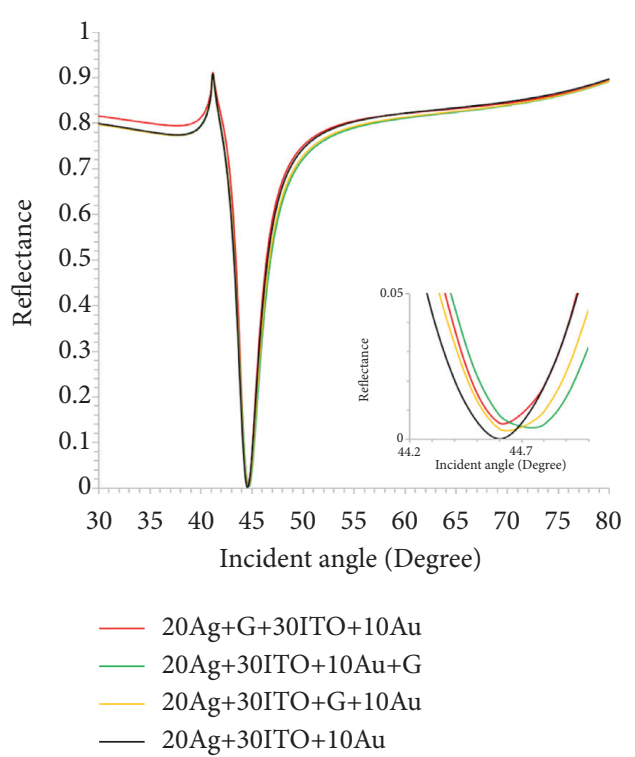

(a)

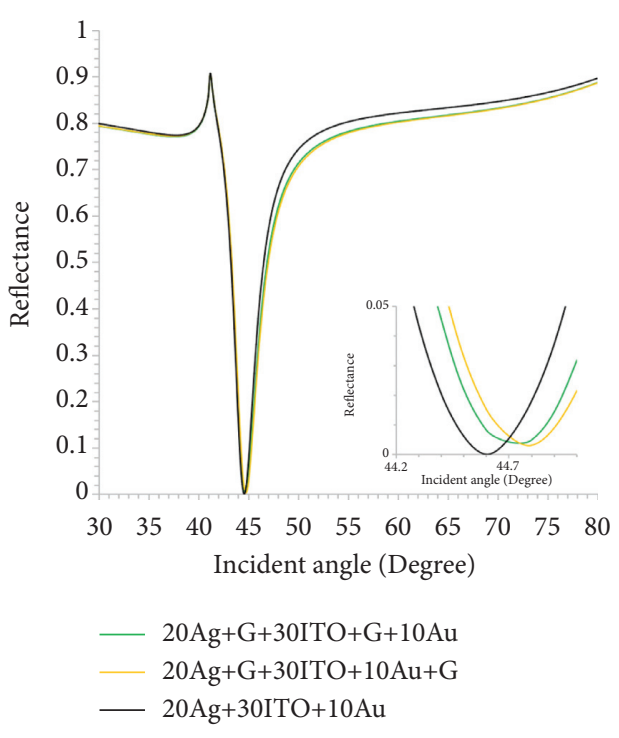

(b)

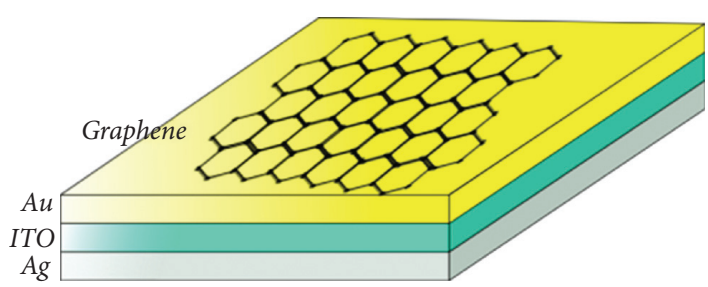

(c)

Figure 5: Sensor reflectivity after graphene was added. (a) A graphene monolayer was added at different positions in the sensor; (b) doublelayer graphene was added at different positions in the sensor (unit: $\mathrm{nm}$ ).

represents the original structure without graphene. Clearly, the structure with the double-graphene layer shows a higher FWHM than that of the original structure. However, a single-graphene layer proved more effective. For structures $\mathrm{Ag} / \mathrm{ITO} / \mathrm{Au} / G, \mathrm{Ag} / \mathrm{ITO} / G / \mathrm{Au}$, and $\mathrm{Ag} / G / \mathrm{ITO} / \mathrm{Au}$, the lowest reflectivities are $0.5 \%\left(\mathrm{FWHM}=2.18, \theta_{\text {res }}=44.8^{\circ}\right)$, $0.4 \%\left(\mathrm{FWHM}=2.09, \theta_{\text {res }}=44.6^{\circ}\right)$, and $0.5 \%(\mathrm{FWHM}=1.75$, $\theta_{\text {res }}=44.6^{\circ}$ ), respectively. All the aforementioned results demonstrate that the addition of a graphene monolayer between the Ag and ITO layers can improve the performance of the structure; although this structure has a higher reflectivity than that of the original one, its FWHM is considerably lower than that of the original structure.

3.3. Effects of Cr Layer on Sensing Chip. For the numerical simulation results to be applicable in actual situations, the influence of a $\mathrm{Cr}$ layer on the sensor performance should be considered. The Cr layer was placed between the glass substrate and the silver layer to enable strong adhesion. The effects of a Cr layer with thicknesses of $0.5-2 \mathrm{~nm}$ on the $\mathrm{Ag} / \mathrm{ITO} / \mathrm{Au}$ and $\mathrm{Ag} / \mathrm{G} / \mathrm{ITO} / \mathrm{Au}$ structures were explored. At $670 \mathrm{~nm}$, the refractive index of $\mathrm{Cr}$ is $3.53+4.27 i$ [10], and we considered this as the reference value.

The effects of different thicknesses of the Cr layer on the sensor are illustrated in Figure 6. Based on the FWHM of the sensor and the minimum reflectivity, the thickness correction made to the $\mathrm{Cr}$ layer at $0.5 \mathrm{~nm}$ has the least effect on the sensor. The red line in the figure illustrates the influence of the $\mathrm{Cr}$ layer on the sensor structure without and with graphene; the minimum reflectances are $0.3 \%$ $\left(\mathrm{FWHM}=2.02, \quad \theta_{\text {res }}=44.6^{\circ}\right)$ and $0.7 \% \quad(\mathrm{FWHM}=1.83$, $\theta_{\text {res }}=44.6^{\circ}$ ), respectively. The data show that the $0.5 \mathrm{~nm} \mathrm{Cr}$ layer in the sensor structure without graphene considerably changed the reflectivity of the sensor. The minimum reflectivity increased by $0.08 \%$ to $0.3 \%$. However, the $\mathrm{Cr}$ layer negligibly affected the FWHMs of the other sensor structures.

3.4. Performance Parameters of SPR Sensor. We analysed the changes in refractive index of biological samples using the following four chip structures: $20 \mathrm{~nm} \mathrm{Ag} / 30 \mathrm{~nm} \mathrm{ITO} / 10 \mathrm{~nm} \mathrm{Au}$; $20 \mathrm{~nm} \mathrm{Ag} / \mathrm{G} / 30 \mathrm{~nm}$ ITO/10 nm Au; $0.5 \mathrm{~nm} \mathrm{Cr} / 20 \mathrm{~nm} \mathrm{Ag} / 30 \mathrm{~nm}$ ITO $/ 10 \mathrm{~nm} \mathrm{Au}$; and $0.5 \mathrm{~nm} \mathrm{Cr} / 20 \mathrm{~nm} \mathrm{Ag} / G / 30 \mathrm{~nm} \mathrm{ITO} / 10 \mathrm{~nm}$ $\mathrm{Au}$. These structures were comprehensively evaluated based on their sensitivities and FOM values. The refractive index of the biological sample layer is defined as $n_{s}=1.33+\Delta n$, where $\Delta n$ is the change in the refractive index of the biological medium. Changes in sensor signals were measured as the refractive index varied from 1.3360 to 1.3405 $(\Delta n=0.0045)$.

The refractive index changes occurring in the four chip structures with respect to the sensing layer are presented in 


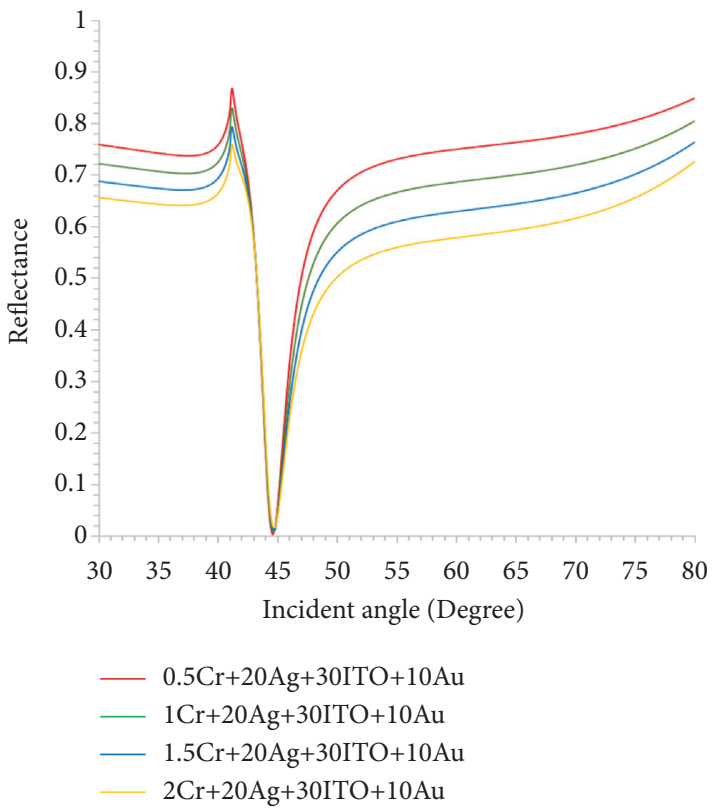

(a)

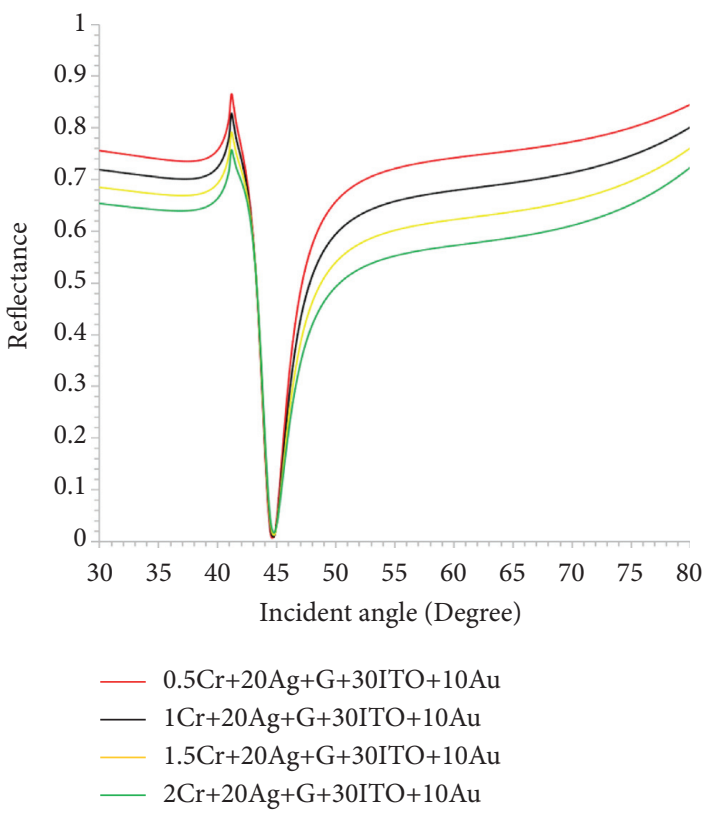

(b)

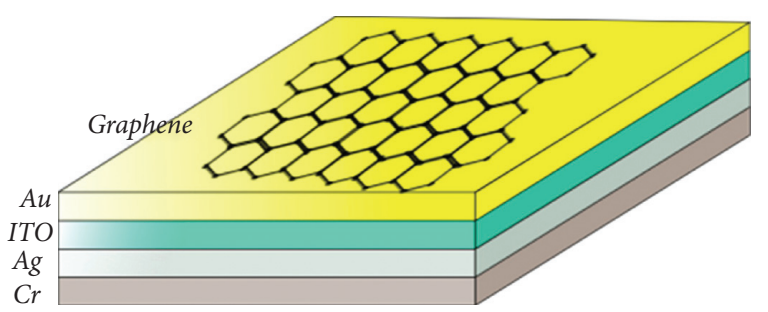

(c)

Figure 6: Reflectivity of two sensors with the Cr layer thickness ranging from 0.5 to 2 (unit: $\mathrm{nm}$ ).
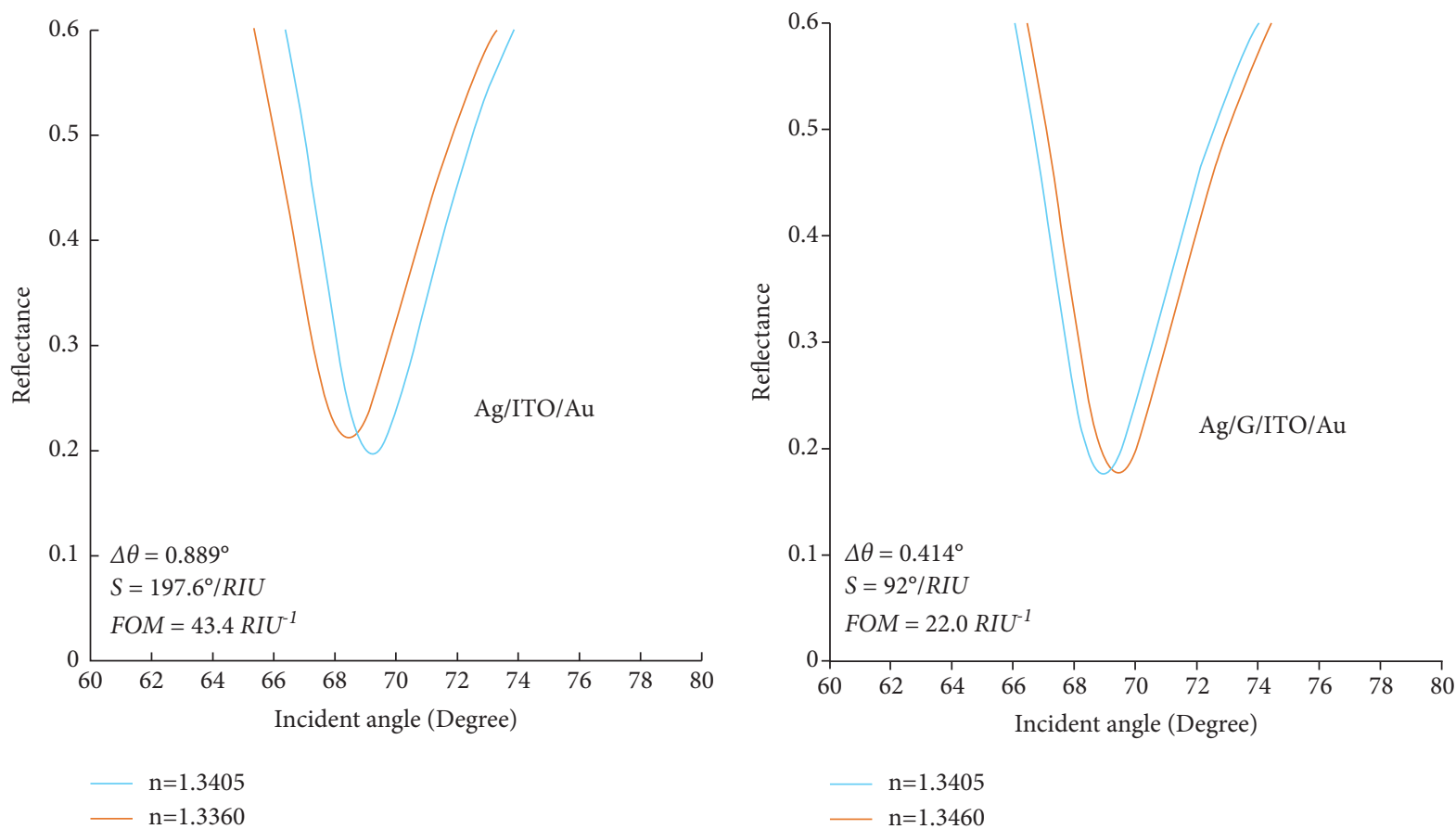

(a)

(b)

Figure 7: Continued. 


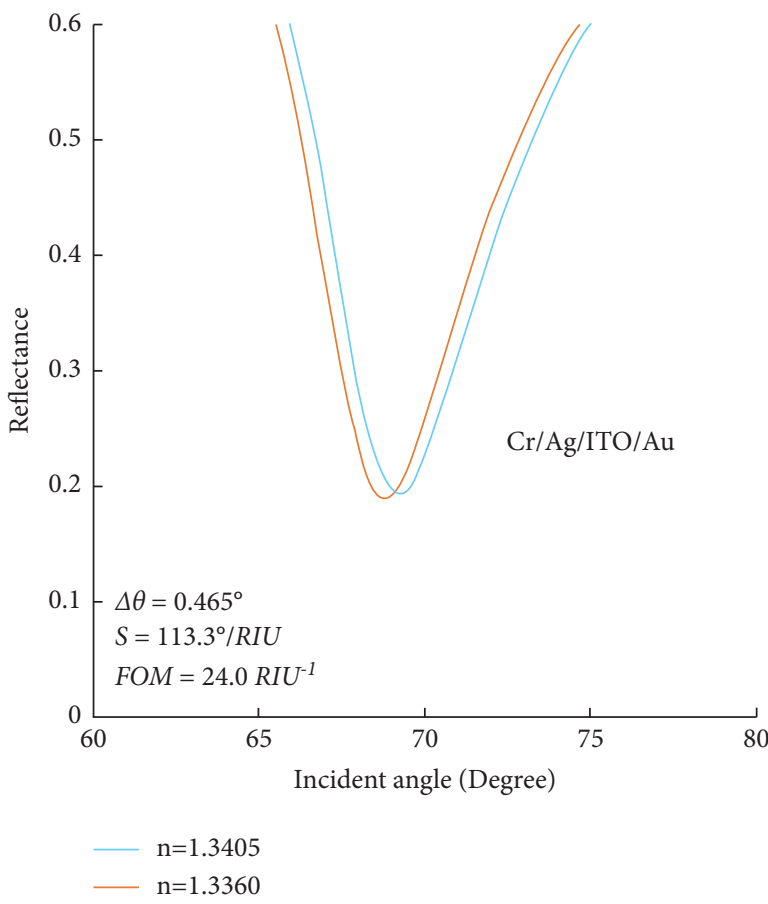

(c)

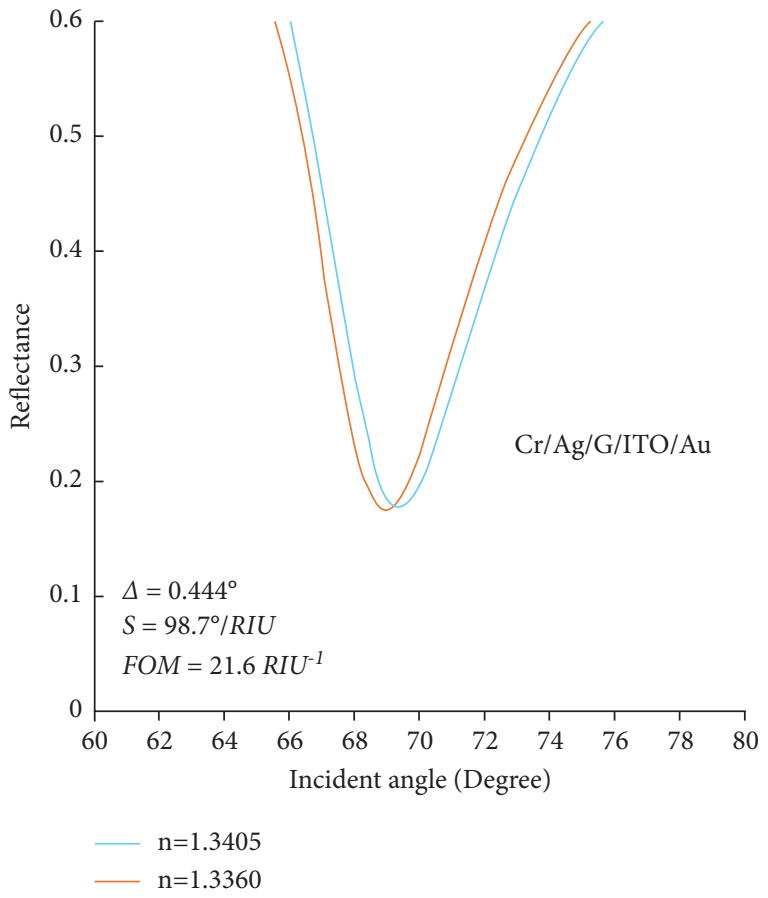

(d)

Figure 7: Changes in reflectance in terms of resonance angles, sensitivities, and FOM values. (a) Ag/ITO/Au; (b) Ag/G/ITO/Au; (c) Cr/Ag/ ITO/Au; and (d) Cr/Ag/G/ITO/Au. $\Delta(n)=0.0045$.

TABLE 2: The influence of the graphene and Cr layer on sensor sensitivity and FOM value.

\begin{tabular}{lccr}
\hline Sensor configuration & Sensitivity & FWHM & FOM \\
\hline $20 \mathrm{~nm} \mathrm{Ag} / 30 \mathrm{~nm} \mathrm{ITO} / 10 \mathrm{~nm} \mathrm{Au}$ & $197.6^{\circ} \mathrm{RIU}^{-1}$ & 4.55 & $43.4 \mathrm{RIU}^{-1}$ \\
$20 \mathrm{~nm} \mathrm{Ag} / \mathrm{G} / 30 \mathrm{~nm} \mathrm{ITO} / 10 \mathrm{~nm} \mathrm{Au}$ & $92^{\circ} \mathrm{RIU}^{-1}$ & 4.19 & $2.0 \mathrm{RIU}^{-1}$ \\
$0.5 \mathrm{~nm} \mathrm{Cr} / 20 \mathrm{~nm} \mathrm{Ag} / 30 \mathrm{~nm} \mathrm{ITO} / 10 \mathrm{~nm} \mathrm{Au}$ & $113.3^{\circ} \mathrm{RIU}^{-1}$ & 4.73 & $24.0 \mathrm{RIU}^{-1}$ \\
$0.5 \mathrm{~nm} \mathrm{Cr} / 20 \mathrm{~nm} \mathrm{Ag} / \mathrm{G} / 30 \mathrm{~nm} \mathrm{ITO} / 10 \mathrm{~nm} \mathrm{Au}$ & $98.7^{\circ} \mathrm{RIU}^{-1}$ & 4.58 & $21.6 \mathrm{RIU}^{-1}$ \\
\hline
\end{tabular}

Figure 7. The sensitivities of $20 \mathrm{~nm} \mathrm{Ag/30} \mathrm{nm} \mathrm{ITO/10} \mathrm{nm} \mathrm{Au}$ and $20 \mathrm{~nm} \mathrm{Ag/G/30} \mathrm{nm} \mathrm{ITO/10} \mathrm{nm} \mathrm{Au} \mathrm{without} \mathrm{the} \mathrm{Cr} \mathrm{layer}$ were $197.6^{\circ} \mathrm{RIU}^{-1}\left(\mathrm{FWHM}=4.55, \mathrm{FOM}=43.4 \mathrm{RIU}^{-1}\right)$ and $92^{\circ} \mathrm{RIU}^{-1}\left(\mathrm{FWHM}=4.19, \mathrm{FOM}=22.0 \mathrm{RIU}^{-1}\right)$, respectively. The sensitivities of the other two structures with a $0.5 \mathrm{~nm}$ stable $\mathrm{Cr}$ layer were $113.3^{\circ} \mathrm{RIU}^{-1} \quad(\mathrm{FWHM}=4.73$, $\left.\mathrm{FOM}=24.0 \mathrm{RIU}^{-1}\right) \quad$ and $98.7^{\circ} \mathrm{RIU}^{-1} \quad(\mathrm{FWHM}=4.58$, $\left.F O M=21.6 \mathrm{RIU}^{-1}\right)$, respectively. We show the change in sensitivity in Table 2. Therefore, in the absence of the $\mathrm{Cr}$ layer, the presence of graphene did not increase the sensitivity of the sensor, thus contradicting our expectations; rather, it slightly reduced the FWHM. This may be because graphene changes the propagation coefficient of the surface plasma wave, causing the light waves concentrated in the Ag or the ITO layer to resonate. Thus, adding graphene reduces the influence of the outermost Au layer such that the response of the outermost metal layer to the change in the external refractive index is reduced. This balance was broken by the addition of the $\mathrm{Cr}$ layer because of which more light waves could resonate with the outermost Au film. However, because of the high refractive index of the $\mathrm{Cr}$ layer, the overall sensitivity of the chip reduced. Nevertheless, the sensitivity of the Ag/ITO/Au chip structure proposed in this
TABle 3: Performance comparison of several reported sensors.

\begin{tabular}{lccc}
\hline Sensor configuration & Sensitivity & $R_{\min }$ & $\mathrm{FOM}$ \\
\hline $\mathrm{Ag} / \mathrm{ITO} / \mathrm{Au}$ & $197.6^{\circ} \mathrm{RIU}^{-1}$ & 0.0008 & $43.4 \mathrm{RIU}^{-1}$ \\
$\mathrm{Ag} / \mathrm{Au}[16]$ & $57.8^{\circ} \mathrm{RIU}^{-1}$ & 0.8 & $56.9 \mathrm{RIU}^{-1}$ \\
$\mathrm{Cr} / \mathrm{Ag}[10]$ & $69.88^{\circ} \mathrm{RIU}^{-1}$ & 0.12 & $15.19 \mathrm{RIU}^{-1}$ \\
$\mathrm{Cr} / \mathrm{Ag} / \mathrm{ITO}[10]$ & $68.77^{\circ} \mathrm{RIU}^{-1}$ & 0.0903 & $11.26 \mathrm{RIU}^{-1}$ \\
$\mathrm{Ag} / \mathrm{Si} / \mathrm{BaTiO}{ }_{3}[17]$ & $130.3^{\circ} \mathrm{RIU}^{-1}$ & 0.01587 & $11.08 \mathrm{RIU}^{-1}$ \\
$\mathrm{Au} / G[17]$ & $53.71^{\circ} \mathrm{RIU}^{-1}$ & 0.125 & $10.74 \mathrm{RIU}^{-1}$ \\
$\mathrm{WS} 2 / \mathrm{G} / \mathrm{Al}[11]$ & $315.5^{\circ} \mathrm{RIU}^{-1}$ & Not reported & $18.8 \mathrm{RIU}^{-1}$
\end{tabular}

paper is still nearly three times higher than that of the Ag/ ITO chip structure proposed by Gan et al. [10], including the sensitivity in terms of the FOM value. In Table 3, we compare the performance of several sensors. The results obtained in this study prove that this type of chip-capacitor SPR sensor can offer significantly high sensitivity compared to that of traditional $\mathrm{Au}$ and $\mathrm{Ag}$ sensors. At the same time, for this structure, the performance enhancement achieved using a semiconductor conductive material such as ITO is better than that achieved by adding graphene, which also provides a new idea for eliminating the effect of graphene monolayer quality problems in practical applications. 


\section{Conclusions}

In summary, variations in the performance of SPR sensing chips following the addition of the semiconductor conductive ITO glass layer between the $\mathrm{Ag}$ and $\mathrm{Au}$ layers were investigated in this study. The performance of the $\mathrm{Ag} / \mathrm{ITO} / \mathrm{Au}$ composite chip was analysed and numerically simulated, and the influence of the graphene and $\mathrm{Cr}$ layers on the chip performance was explored. Finally, the sensitivity and FOM for each chip structure were obtained via simulations involving changes in the refractive index of the biosensitive layer. Numerical simulation results showed that the structure comprising $20 \mathrm{~nm} \mathrm{Ag}, 30 \mathrm{~nm}$ ITO, and $10 \mathrm{~nm}$ $\mathrm{Au}$ exhibited the best performance; the angular sensitivity was $197.6^{\circ} \mathrm{RIU}^{-1}$; and the FOM was $43.4 \mathrm{RIU}^{-1}$. Although the sensor structure exhibits good performance, we still need to determine methods for accurately controlling the thickness of the sensing layer during actual manufacturing. This type of biosensor, wherein the sensitivity is improved through structural changes, is expected to reduce dependence on some photosensitive materials, thereby facilitating easy and economical biological sensing processes. The findings of this study can serve as a theoretical basis for improving the sensitivity of SPR sensors via structural changes.

\section{Data Availability}

The data used to support the findings of this study are included within the article.

\section{Conflicts of Interest}

The authors declare no conflicts of interest.

\section{Acknowledgments}

This research was supported by the National Natural Science Foundation of China (Grant no. 81703271), National Key Research and Development Program of China (Grant nos. 2018YFC0809200 and 2016YFF0203203), Guangdong Science and Technology Foundation (Grant nos. 2020B1111160001 and 2017B020210006), Shenzhen Science and Technology Foundation (Grant nos. GJHZ20200731095604013, JSGG20200807171602031, 201906133000069, and SGLH20180625171602058), and Open Project of Key Laboratory of Tropical Disease Control of Ministry of Education (Sun Yatsen University) (Grant no. 2019kfkt06).

\section{References}

[1] Y. Zeng, L. Wang, S.-Y. Wu et al., "Wavelength-scanning SPR imaging sensors based on an acousto-optic tunable filter and a white light laser," Sensors, vol. 17, no. 1, p. 90, 2017.

[2] Y. Zeng, R. Hu, L. Wang et al., "Recent advances in surface plasmon resonance imaging: detection speed, sensitivity, and portability," Nanophotonics, vol. 6, no. 5, pp. 1017-1030, 2017.

[3] E. Wijaya, C. Lenaerts, S. Maricot et al., "Surface plasmon resonance-based biosensors: from the development of different SPR structures to novel surface functionalization strategies," Current Opinion in Solid State and Materials Science, vol. 15, no. 5, pp. 208-224, 2011.

[4] S. Zeng, K. V. Sreekanth, J. Shang et al., "Graphene-gold metasurface architectures for ultrasensitive plasmonic biosensing," Advanced Materials, vol. 27, no. 40, pp. 6163-6169, 2015.

[5] S. Szunerits, N. Maalouli, E. Wijaya, J.-P. Vilcot, and R. Boukherroub, "Recent advances in the development of graphene-based surface plasmon resonance (SPR) interfaces," Analytical and Bioanalytical Chemistry, vol. 405, no. 5, pp. 1435-1443, 2013.

[6] A. S. Kushwaha, A. Kumar, R. Kumar, and S. K. Srivastava, "A study of surface plasmon resonance (SPR) based biosensor with improved sensitivity," Photonics and Nanostructures-Fundamentals and Applications, vol. 31, pp. 99106, 2018.

[7] H. Y. Cai, D. Cui, and L. Zhang, "Surface plasmon resonance characteristic study of graphene-on-gold structure," in Proceedings of the 12th IEEE international conference on nanotechnology (IEEE-NANO) (2012 20-23 Aug.), Birmingham, England, August 2012.

[8] R. Verma, B. D. Gupta, and R. Jha, "Sensitivity enhancement of a surface plasmon resonance based biomolecules sensor using graphene and silicon layers," Sensors and Actuators B: Chemical, vol. 160, no. 1, pp. 623-631, 2011.

[9] X. Zhao, T. Huang, P. Ping et al., "Sensitivity enhancement in surface plasmon resonance biochemical sensor based on transition metal dichalcogenides/graphene heterostructure," Sensors, vol. 18, no. 7, pp. 2056-2066, 2018.

[10] S. M. Gan, P. S. Menon, N. R. Mohamad, N. A. Jamil, and B. Y. Majlis, "FDTD simulation of Kretschmann based Cr-AgITO SPR for refractive index sensor," Materials Today: Proceedings, vol. 7, pp. 668-674, 2019.

[11] L. Wang, Y. Sun, J. Wang et al., "Sensitivity enhancement of SPR biosensor with silver mirror reaction on the Ag/Au film," Talanta, vol. 78, no. 1, pp. 265-269, 2009.

[12] J. Qu, J. Wang, H. Zhang et al., "Toxicokinetics and systematic responses of differently sized indium tin oxide (ITO) particles in mice via oropharyngeal aspiration exposure," Environmental Pollution, vol. 290, Article ID 117993, 2021.

[13] B. Ruan, Q. You, J. Zhu et al., "Improving the performance of an SPR biosensor using long-range surface plasmon of Gadoped zinc oxide," Sensors, vol. 18, no. 7, pp. 2098-2106, 2018.

[14] C. Rizal, S. Pisana, and I. Hrvoic, "Improved magneto-optic surface plasmon resonance biosensors," Photonics, vol. 5, no. 3, pp. 15-31, 2018.

[15] J. Maurya, A. François, and Y. Prajapati, "Two-dimensional layered nanomaterial-based one-dimensional photonic crystal refractive index sensor," Sensors, vol. 18, no. 3, pp. 857-864, 2018.

[16] S. H. Kim, T. U. Kim, H. Y. Jung, H. C. Ki, D. G. Kim, and B.-T. Lee, "The effect of $\mathrm{Au} / \mathrm{Ag}$ bimetallic thin-films on surface plasmon resonance properties comparing with those of Au and Ag single thin-films," Journal of Nanoscience and Nanotechnology, vol. 18, no. 3, pp. 1777-1781, 2018.

[17] S. M. A. Uddin, S. S. Chowdhury, and E. Kabir, "Numerical analysis of a highly sensitive surface plasmon resonance sensor for SARS-CoV-2 detection," Plasmonics, vol. 16, no. 6, pp. 2025-2037, 2021.

[18] S. J. Rowley-Neale, E. P. Randviir, A. S. Abo Dena, and C. E. Banks, "An overview of recent applications of reduced 
graphene oxide as a basis of electroanalytical sensing platforms," Applied Materials Today, vol. 10, pp. 218-226, 2018.

[19] B. A. Prabowo, A. Alom, M. K. Secario et al., "Graphene-based portable SPR sensor for the detection of Mycobacterium tuberculosis DNA strain," Procedia Engineering, vol. 168, pp. 541-545, 2016.

[20] A. S. Kushwaha, A. Kumar, R. Kumar, M. Srivastava, and S. K. Srivastava, "Zinc oxide, gold and graphene-based surface plasmon resonance (SPR) biosensor for detection of pseudomonas like bacteria: a comparative study," Optik, vol. 172, pp. 697-707, 2018.

[21] W. Wei, J. Nong, Y. Mei, C. Zhong, G. Lan, and W. Hu, "Single-layer graphene-coated gold chip for enhanced SPR imaging immunoassay," Sensors and Actuators B: Chemical, vol. 273, pp. 1548-1555, 2018. 